Les sources de l'Histoire des Mines : Nouveaux outils, Nouvelles approches

\title{
Une tentative d'inventaire des richesses minières de la France : l'enquête du Régent, 1716-1718
}

An attempt to inventory the mineral riches of France: the Régent's survey, 1716-1718

\section{Christiane Demeulenaere-Douyère}

\section{OpenEdition \\ Journals}

Édition électronique

URL : http://journals.openedition.org/dht/753

DOI : $10.4000 /$ dht.753

ISSN : $1775-4194$

\section{Éditeur :}

Centre d'histoire des techniques et de l'environnement du Cnam (CDHTE-Cnam), Société des élèves du CDHTE-Cnam

\section{Édition imprimée}

Date de publication : 1 décembre 2008

Pagination : $9-16$

ISBN : 978-2-95-30779-2-6

ISSN : 0417-8726

\section{Référence électronique}

Christiane Demeulenaere-Douyère, « Une tentative d'inventaire des richesses minières de la France : l'enquête du Régent, 1716-1718», Documents pour l'histoire des techniques [En ligne], 16 | $2^{2}$ semestre 2008, mis en ligne le 08 novembre 2010, consulté le 08 septembre 2020. URL : http:// journals.openedition.org/dht/753; DOI : https://doi.org/10.4000/dht.753 


\title{
Une tentative d'inventaire des richesses minières de la France : l'enquête du Régent, 1716-1718
}

\author{
Christiane Demeulenaere-Douyère \\ Archives nationales, site de Paris \\ CDHTE-Cnam
}

\begin{abstract}
RÉSUMÉ
À la fin de 1715, Philippe d'Orléans, Régent de France, lance une grande enquête nationale pour collecter des renseignements à la fois sur la qualité et la quantité des ressources naturelles dont le royaume peut disposer pour restaurer sa situation économique, catastrophique à la fin du règne de Louis XIV. Dans cet immense chantier qui va durer trois années, sont impliqués le pouvoir royal en la personne du Régent et de son conseiller l'abbé Bignon qui inspirent les lignes directrices de l'enquête, les intendants et leurs subdélégués qui vont chercher informations et échantillons au plus profond des provinces, et aussi, ce qui est nouveau, l'Académie royale des sciences qui, en la personne de Réaumur, assure la direction scientifique de l'enquête et apporte son expertise à l'analyse des résultats.

Les documents provenant de l'enquête, conservés aujourd'hui aux Archives de l'Académie des sciences et jusqu'à présent insuffisamment exploités, peuvent apporter beaucoup à l'histoire minière de la France pré-industrielle par l'abondance et la précision des informations qu'ils fournissent sur la diversité des minerais exploitables dans le royaume, sur les conditions de leur exploitation, l'outillage et même les mineurs.
\end{abstract}

Résumés et mots clés en anglais sont regroupés en fin de volume, accompagnés des mots clés français

$\mathrm{O}$ trouve aux Archives de l'Académie des sciences trois cartons au titre énigmatique : « Enquêtes du Régent ॥. Ces documents, et d'autres dispersés dans divers dossiers, sont le résultat d'une grande enquête réalisée entre 1716 et 1718 dans l'ensemble du royaume, sous les ordres de Philippe d'Orléans, alors Régent de France, et sous la direction scientifique de l'Académie royale des sciences. Lors de cette enquête dont le but officiel était de constater l'état des richesses naturelles - et particulièrement minérales - de la France, le Régent a transmis pour examen à l'Académie des sciences les mémoires, lettres et autres documents qu'il recevait des intendants ; c'est dans les archives de cette institution qu'ils se trouvent encore.

L'enquête de 1716-1718 qui est caractérisée par une remarquable collaboration entre l'État et l'Académie royale des sciences, est restée peu ou pas connue de la plupart des historiens', même si les documents qui nous en sont parvenus ont été signalés dans le guide des Archives de l'Académie des sciences ${ }^{2}$ et si, au colloque organisé en 1999 pour commémorer le troisième centenaire de la réforme de l'Académie en 1699, une communication en a esquissé les grandes

\footnotetext{
1 Dans Les sources statistiques de l'histoire de France (Genève-Paris, Droz, 1964, 2e éd., 1980), Bertrand Gille semble ignorer l'enquête du Régent lorsqu'il traite des enquêtes consacrées aux mines ou à la métallurgie sous l'Ancien Régime (pp. 62-67), pour l'évoquer curieusement au titre des enquêtes agricoles (p. 71), citant I'article de Jean Pilisi, "L'enquête ordonnée par le régent sur les richesses naturelles de la France (1716-1717) 11, Revue d'histoire des sciences, XVI, 1963, pp. 373-374.

2 Éric Brian et Christiane Demeulenaere-Douyère dir., Histoire et mémoire de l'Académie des sciences. Guide de recherches, Paris, Tec \& Doc, 1996, p. 66.
} 


\section{L'enquête du Régent, 1716-1718}

lignes ${ }^{3}$. Hormis quelques érudits qui ont parfois utilisé ces documents pour étudier l'histoire d'une région ou d'une localité, ils n'ont jamais été jusqu'à présent analysés dans leur ensemble. Pourtant leur intérêt est majeur et multiple, car, au-delà de la seule histoire régionale, ils ont à apporter à l'histoire politique, économique et sociale, à l'histoire des sciences et, bien sûr, à l'histoire de l'Académie royale des sciences. Aussi en avons-nous, avec le Pr. David J. Sturdy, professeur d'histoire émérite de l'Université d'Ulster (Coleraine, Irlande du Nord), préparé la publication afin de les mettre plus largement à la disposition des chercheurs dans une édition critique ${ }^{4}$.

\section{Les documents de l'enquête}

La plupart des documents relatifs à l'enquête du Régent se trouvent dans trois cartons de la série des « pochettes de séances ॥ des archives de l'Académie des sciences, intitulés "Enquêtes du Régent».

L'enquête ayant été menée à l'initiative du gouvernement royal, il aurait été logique - et conforme à nos usages archivistiques - que ces documents soient aujourd'hui conservés aux Archives nationales, avec d'autres correspondances adressées au gouvernement par les intendants. Toutefois, le rôle central joué par l'Académie des sciences et particulièrement par Réaumur dans la direction de l'enquête explique aisément pourquoi ces documents sont demeurés dans les archives de cette institution.

II s'agit de lettres, de mémoires et de papiers de nature très variée : correspondance entre le Régent, les intendants et l'Académie des sciences, ou entre les intendants et leurs « informateurs » locaux ; mémoires et rapports envoyés par les intendants; réponses de l'Académie des sciences et demandes de renseignements complémentaires; correspondance entre le Régent (ou l'Académie) et certains particuliers qui sollicitent des privilèges ou proposent des informations sur des mines ou d'autres sources de minerai ; rapports des analyses effectuées dans le laboratoire de l'Académie ; correspondance enfin entre Bignon et le Régent ou quelquefois des ministres du roi. Il y a aussi

3 David J. Sturdy, " L'Académie royale des sciences et l'enquête du Régent de 1716-1718 », dans Christiane Demeulenaere-Douyère et Éric Brian éd., Règlement, usages et science dans la France de l'absolutisme : à l'occasion du troisième centenaire du règlement instituant l'Académie royale des sciences (26 janvier 1699), Paris, Tec \& Doc, 2002, pp. 138-139.

4 Christiane Demeulenaere-Douyère et David J. Sturdy, L'enquête du Régent, 1716-1718. Sciences, techniques et politique dans la France préindustrielle, Turnhout, Brépols, 2008. des documents qui ne font pas partie de l'enquête et dont la présence dans ces cartons relève, semble-t-il, du hasard.

Il est difficile de préciser le nombre exact des documents, car, mêlés aux mémoires et aux correspondances, il y a aussi des notes dont la relation avec l'enquête pose problème. On peut toutefois avancer un total d'environ 550 documents, auxquels il faut ajouter environ 130 documents provenant du fonds Réaumur des Archives de l'Académie des sciences lessentiellement des minutes d'observations et de demandes d'éclaircissements rédigées par Réaumur lui-même) ${ }^{5}$ et quelques documents provenant des pochettes de séances des années 1715 à 1718. Soit au total un corpus d'environ 700 documents.

Pour rendre plus intelligible la présentation de cette abondante documentation, nous l'avons organisée en trois parties. La première, relativement courte, est composée des quelques documents relatifs aux origines de l'enquête dont on dispose. La deuxième, qui est de loin la plus volumineuse, reprend tous les documents rassemblés par l'enquête en les classant par généralité ou province ; à l'intérieur de chacune, ils sont présentés chronologiquement dans la mesure où ils sont datés ou selon que les sujets dont ils traitent permettent de les relier logiquement les uns aux autres. La troisième partie rassemble les procès-verbaux et commentaires rédigés par Réaumur et un certain Fousjean à la suite des essais effectués en laboratoire sur les échantillons de minerais envoyés par les intendants ${ }^{6}$.

Aussi nombreux qu'ils soient, il est évident que les documents dont nous disposons aujourd'hui sur l'enquête du Régent n'en constituent pas la totalité. On trouve des références à des lettres ou à des mémoires complémentaires qui manquent aujourd'hui à l'Académie des sciences. Particulièrement, le nombre des dessins et des plans aujourd'hui conservés est très inférieur à ce qu'il devait être à l'issue de l'enquête ${ }^{7}$. Ces lacunes ne sont pas étonnantes, en raison du nombre important de personnes qui ont contribué à l'enquê-

5 Arch. Académie des sciences, Paris, fonds Réaumur, carton 6 ; ces documents épars ont été rassemblés par nos soins dans le dossier 2 et numérotés sans reclassement préalable de 1 à 126.

6 Arch. Académie des sciences, Paris, carton 17 « Enquêtes du Régent 1 .

7 Sur ces questions, Christiane Demeulenaere-Douyère, « Enquête administrative et dessin technique dans la France préindustrielle : l'exemple de l'enquête du Régent, 1716-1718 "l, dans Actes du $132^{\circ}$ congrès national des sociétés historiques et scientifiques, tenu à Arles en avril 2007, Paris, Éd. du CTHS, à paraître. 


\section{Christiane Demeulenaere-Douyère}

te et de la masse de la documentation rassemblée. Une autre hypothèse est que Bignon et Réaumur, qui avaient accès à ces documents, en ont conservé certains par devers eux et que, dans bien des cas, ceux-ci ont ensuite disparu. C'est très probablement ce qui s'est produit avec Réaumur qui a sans doute mis de côté des mémoires et des documents iconographiques provenant de l'enquête pour son autre entreprise, les Descriptions des arts et métiers ; à sa mort, il a légué tous ses papiers à l'Académie des sciences pour que ses collègues puissent poursuivre cette œuvre, mais on sait que les académiciens se sont partagés ces papiers et que " tout a été au pillage $\|^{8}$, sans que rien n'en ait jamais été publié. Enfin, une autre explication tout aussi plausible est que, sous l'Ancien Régime, les archives de l'Académie n'étaient pas classées selon un système spécifique et que certains documents ont été depuis dispersés ou perdus?

Enfin, il est certain aussi que d'autres documents relatifs à l'enquête de 1716-1718 sont aujourd'hui conservés hors de Paris, et peut-être même hors de France. On en a localisés à la Bibliothèque municipale de Besançon et aux Archives municipales de Strasbourg, ainsi qu'aux Archives départementales des Pyrénées-Orientales. L'édition que nous avons donnée, ne prétend donc pas à embrasser l'intégralité des documents de l'enquête du Régent, ce qui serait sans doute une tentative sans espoir.

\section{La stratégie d'une enquête administrative}

Une des originalités majeures que présentent les archives de l'enquête sur les richesses naturelles de la France de 1716-1718 est qu'elles permettent d'étudier pratiquement in vivo le processus d'une enquête officielle au début du $\mathrm{xvII}{ }^{\mathrm{e}}$ siècle et la manière dont le pouvoir central se constitue un savoir administratif. Plus encore, ces documents n'ayant pas été finalisés dans un rapport définitif, ils ont conservé leurs accents authentiques et restituent une " parole » que la pratique administrative a tendance à banaliser dans un discours formel.

8 Christiane Demeulenaere-Douyère, " Des 'papiers' de I'Académie des sciences à ses archives ", dans C. Demeulenaere-Douyère et É. Brian éd., Règlement, usages et science..., op. cit. note 3, p. 473.

9 Christiane Demeulenaere-Douyère, « Esquisse d'une histoire des archives de l'Académie des sciences ॥, dans É. Brian et $\mathrm{C}$. Demeulenaere-Douyère dir., Histoire et mémoire de l'Académie des sciences, op. cit. note 2, pp. 45-54.
Trois acteurs majeurs

L'enquête est l'œuvre de trois hommes.

En premier lieu, Philippe d'Orléans, Régent de France ${ }^{10}$, qui, au-delà de sa sulfureuse réputation de libertin, est un homme éclairé, ouvert aux sciences. II a été initié, à partir de 1702, à la chimie par Guillaume Homberg. Ce dernier, convaincu de l'utilité des sciences et de leur nécessaire application à la solution de questions pratiques, a converti son illustre élève à ses vues $^{11}$. Sans doute son influence a-t-elle lourdement pesé dans la décision de mettre en œuvre cette enquête.

À ses côtés, il y a son conseiller, l'abbé Jean-Paul Bignon. Il est le très brillant neveu de Louis Phélypeaux, comte de Pontchartrain, protecteur de l'Académie royale des sciences depuis la mort de Louvois en 1691 , qu'il représente auprès de l'Académie. C'est lui qui a préparé et mis en œuvre la réforme de 1699 qui a "renouvellé » l'ancienne Académie des sciences fondée en 1666.

Le troisième homme est un jeune géomètre, RenéAntoine Ferchault de Réaumur (1683-1757) 12. Remarqué par Guisnée, puis par Varignon qui l'introduit à l'Académie des sciences en 1708 comme son élève, Réaumur se voit confier la direction des Descriptions des arts et métiers quand, en 1711, il est promu au rang de pensionnaire de l'Académie. II s'agit d'un projet ancien de publication collective que l'Académie traîne depuis les premiers temps de l'ancienne Académie (1675) et qui n'avance guère. Ouvrage utilitaire à caractère encyclopédique, il devra réunir des savoirs et des savoir-faire sur les arts mécaniques, avec le but d'en vulgariser l'usage auprès des artisans. Réaumur va trouver d'autant plus d'intérêt à l'enquête de 1716-1718 qu'elle va être aussi pour lui un moyen de réunir rapidement les informations nécessaires à la rédaction des Descriptions des arts et métiers.

10 Sur le Régent, voir Jean-Christian Petitfils, Le Régent, Paris, Fayard, 1996, et Denis Reynau et Chantal Thomas éd., Le Régent : entre fable et histoire, Paris, CNRS, 2003.

11 Sur cet aspect de la culture du Régent, voir C. Demeulenaere-Douyère et D. J. Sturdy, L'enquête du Régent, op. cit. note 4, Introduction, et Simone Mazauric, « Philippe d'Orléans et les sciences ॥, Cahiers Saint-Simon, n. 34, 2006.

12 Sur Réaumur, voir son éloge par Grandjean de Fouchy, HMARS, 1757, pp. 201-206, et notice dans Charles C. Gillispie éd., Dictionary of scientific biography, New York, Scribner, 1970-1990 ; aussi, Jean Torlais, Réaumur : Un esprit encyclopédique en dehors de "L'Encyclopédie ". D'après des documents inédits, Paris, A. Blanchard, 1961, et Gilles Bresson, Réaumur : le savant qui osa croiser une poule avec un lapin, Château d'Olonne, d'Orbestier, 2001. 


\section{L'enquête du Régent, 1716-1718}

Un autre élément déterminant dans l'organisation de l'enquête de 1716-1718 est constitué par le précédent de l'enquête dirigée par le duc de Beauvillier " pour l'instruction du duc de Bourgogne » en 1697. Pour réaliser cette enquête, Beauvillier qui agissait lui aussi au nom du roi, avait adressé aux intendants un mémoire précis les obligeant à donner certaines informations sur les généralités. Ils devaient, par exemple, vérifier les cartes des provinces, aborder le gouvernement militaire, la justice, les affaires de l'église, le commerce, l'industrie, les finances, les tailles et les droits des aides ${ }^{13}$. Pendant deux années, les intendants ont rédigé des réponses et envoyé à la cour un total de vingt-neuf mémoires ${ }^{14}$.

\section{L'élaboration de l'enquête}

L'intérêt majeur de l'enquête du Régent est la masse documentaire qu'on en a conservée, qui permet d'étudier le détail de son élaboration. Elle révèle une méthode de travail qui met en œuvre à la fois une chaîne interactive d'informateurs et l'expertise de l'Académie royale des sciences.

Dès le début de 1716, les intendants reçoivent un mémoire général qui ne tarde pas à leur être rappelé par des instructions particulières, le plus souvent rédigées par Réaumur. lls doivent se mettre au travail et commencer à préparer leurs réponses. Les demandes étant rédigées au nom du Régent, ils ont l'obligation d'y répondre, mais leur enthousiasme n'est pas toujours sans équivoque ; certains répondent rapidement et reçoivent en retour des réactions de l'Académie dans les six premiers mois de l'année $1716^{15}$, mais d'autres se font tirer l'oreille.

Pour les intendants consciencieux, qui sont la grande majorité, commence alors un travail considérable qui vient s'ajouter à leurs tâches administratives courantes, déjà lourdes. Ils doivent recourir à leur

\footnotetext{
13 Publié dans E. Esmonin, "Les mémoires des intendants pour l'instruction du duc de Bourgogne (étude critique) ", Bulletin de la Société d'histoire moderne, LV, 1956. Sur l'enquête en général, voir B. Gille, Les sources statistiques..., op. cit. note 1, pp. 28-33.

14 Le Comité des travaux historiques et scientifiques (CTHS) a repris activement la publication des mémoires des intendants. Les manuscrits et leurs divers états intermédiaires sont aujourd'hui très dispersés; certaines copies se trouvent aux Archives nationales (Paris), dans la série H. Pour leur localisation plus précise, voir les introductions des diverses éditions qui en ont été données.

15 C. Demeulenaere-Douyère et D. J. Sturdy, L'enquête du Régent, op. cit. note 4, «Rouen », doc. 2.
}

propre réseau de correspondants ${ }^{16}$ pour recueillir les informations que demande l'enquête. En effet, si certains intendants se déplacent en personne, mettant à profit leur tournée dans la province ${ }^{17}$ ou la tenue des États ${ }^{18}$, la plupart s'en rapportent sur le terrain à des " informateurs » qui ont une connaissance plus intime de leur région : des agents investis de fonctions officielles comme les subdélégués ${ }^{19}$, les juges ${ }^{20}$ ou les ingénieurs du roi ${ }^{21}$, des membres de compagnies savantes comme les académies de province ${ }^{22}$ qui se révèlent fort actives, ou de simples particuliers, mineurs, prêtres, militaires ${ }^{23}$, artisans ${ }^{24}$, pêcheurs ${ }^{25}$, etc., soit au total un éventail considérable de personnages qui fournissent aux intendants les renseignements et les échantillons dont ils ont besoin.

Après avoir recueilli les premières données, les intendants rédigent des mémoires qu'ils envoient au Régent, parfois accompagnés d'échantillons de minerais, et même de modèles réduits de certaines installations comme les fours ${ }^{26}$. Le Régent transmet tous ces matériaux à Bignon ou le plus souvent à Réaumur.

Pour les intendants, rassembler les informations demandées n'est pas forcément aisé. D'abord, ils sont tributaires de leurs propres "informateurs ", plus ou moins zélés à les aider rapidement. Certains « infor-

16 Ainsi, quand on le rappelle à l'ordre, l'intendant d'Auch et Béarn répond qu'«il a écrit partout pour avoir les mémoires que désire l'accadémie ; il ne manquera pas de les envoyer quand ils seront rassembléz » (ibid., « Navarre et Béarn », doc. 15).

17 Par exemple, ibid., "Lyon », doc. 7 : « Cependant la nonchalance de ceux auxquels il s'est adressé et le peu de secours qu'on trouve dans la pluspart des personnes de province l'ont déterminé à différer d'envoyer son second mémoire jusques au tems que, parcourant cette généralité pour le département des tailles, il ait pu travailler par luy même à satisfaire aux questions qui luy ont esté faittes par l'accadémie des Sciences. »

18 Ibid., " Navarre et Béarn ॥, doc. 11.

19 Ibid., "Languedoc ", doc. 15.

20 Ibid., " Navarre et Béarn ॥, doc. 3.

21 Ibid., "Bretagne ॥, doc. 7.

22 Ibid., « Bordeaux ou Guyenne », doc. 20.

23 Ibid., « Perpignan », doc. 9.

24 Ibid., "Les analyses de minerais ॥, doc. 3 (Languedoc). 25 Ibid., " Aix-en-Provence ॥, doc. 22.

26 Ainsi, Legendre de Lormoy : " Je prends aussi la liberté de joindre à ma lettre [...] un dessein du four où l'on a fait cuire les pierres. J'en ay aussy fait faire un modèle en carton que j'ay mis dans le balot pour donner à l'académie une connoissance plus exacte de l'intérieur du fourneau. " (ibid., " Navarre et Béarn », doc. 20). Voir aussi ibid., "Lyon », doc. 16. II ne subsiste malheureusement aucun de ces modèles de carton, bois ou plâtre. 
mateurs / sont imprécis ${ }^{27}$, d'autres sont très imaginatifs et donnent des renseignements plus ou moins fantaisistes qu'il faut ensuite aller contrôler sur le terrain ${ }^{28}$. Des obstacles matériels peuvent également ralentir les progrès de l'enquête. Dans les régions montagneuses, les mines dont on veut tirer des échantillons sont souvent difficiles d'accès, situées dans des endroits escarpés, inaccessibles en hiver à cause de la neige ${ }^{29}$, ou elles sont susceptibles d'être inondées ${ }^{30}$ ou d'être soumises à d'autres aléas naturels : ainsi, dans une mine des Pyrénées, des chauves-souris gênent l'accès d'une grotte dont on veut tirer des échantillons ${ }^{31}$. Par ailleurs, alors que la plupart des « informateurs " acceptent d'apporter leur concours à l'enquête sans poser de questions, sinon avec enthousiasme, certains se méfient et refusent de collaborer malgré des injonctions de l'intendant ${ }^{32}$.

Les mémoires adressés à Paris sont souvent accompagnés d'échantillons de matières minérales (or, argent, fer, étain, plomb...), de pierres précieuses ou non (turquoises, améthystes, marbres, craies...), de coraux et autres produits de la mer, et de sables, de perles, d'eaux minérales, voire de pétrole... Les intendants, après les avoir collectés auprès de leurs subdélégués ou autres « informateurs», les emballent le plus soigneusement qu'ils peuvent ${ }^{33}$ et les envoient

27 Pourtant fort zélé à servir l'enquête, l'intendant de Lyon écrit : " Je demande mil excuses à VAR sy j'ay tant tardé à luy envoyer les éclaircissements qu'elle m'a fait l'honneur de me demander sur plusieurs articles du premier mémoire que j'ay pris la liberté de luy adresser pour l'académie des Sciences, mais c'est un ouvrage qui n'a pas dépendu de moy seul. Il a falu tirer des connoissances de différentes personnes et s'assujettir à leur peu d'exactitude. ॥ (Ibid., "Lyon ॥, doc. 7). 28 Ainsi, en Auvergne : «Ces mémoires ont été dressés par un homme de la province, lequel a beaucoup d'esprit et de littérature, mais, comme il ne s'est pas donné la peine de se transporter sur les lieux pour voir et examiner par luy même les choses rares et curieuses qui sont répendues dans son mémoire, on ne peut ajouter une foy entière aux faits qui y sont avancés. ॥ (Ibid., « Riom ou Auvergne ॥, doc. 5).

29 Ibid., « Perpignan », doc. 4.

30 Ibid., "Les analyses de minerais ॥, doc. 1 (Alsace).

31 Ibid., « Navarre et Béarn ॥, doc. 3.

32 Ibid., "Caen ॥, doc. 5-10.

33 Les conditions d'envoi de ces échantillons sont une réelle difficulté que Réaumur lui-même examine dans le détail. Ainsi : « On souhaiteroit avoir de l'huile de Gabian qu'on fust sûr qui n'eût point été mélangée. On en auroit besoing de deux ou trois livres avec son écume. II seroit à propos de la metre dans des bouteilles de grès ou de verre " (ibid., "Languedoc », doc. 4). Ou encore : "À leur arrivée à Marseille, on renfermeroit ces branches [de corail] dans un petit baril de bois ou de fer blanc qu'on rempliroit d'eau de mer et à Paris par les moyens les plus rapides à leur disposition ; nombre de correspondances abordent cette épineuse question.

L'enquête suscite aussi un intérêt plus général parmi les particuliers, surtout chez les exploitants de mines et les fabricants de fer et d'acier, qui espèrent en tirer profit. Le Régent se voit adresser des offres d'informations ou des propositions pour l'exploitation de minerais, moyennant souvent l'octroi d'un privilège, ce qu'il reçoit avec prudence ${ }^{34}$.

\section{L'expertise de l'Académie royale des sciences}

Celle-ci se manifeste dès les premiers moments de l'enquête puisque c'est l'Académie qui élabore, dès la fin de 1715, un «Projet de lettre circulaire de Mgr le duc d'Orléans à MM. les Intendans, pour appuyer les mémoires des recherches que SAR a chargé l'Académie des sciences de suivre dans les différentes provinces du royaume par rapport à la physique et aux arts $\|{ }^{35}$. L'objet de l'enquête y est clairement désigné :

"Comme la perfection des sciences et des arts fait un des principaux soins de Monseigneur le duc d'Orléans, Régent du royaume, et que SAR a recconnu combien les recherches en ce genre peuvent contribuer à faire refleurir la France, [l'Académie] exhorte les François qui sont à portée de contribuer de quelque manière que ce soit, à un dessein si avantageux à leur patrie, de considérer avec attention tout ce que la nature et l'art produisent de plus important dans les régions où ils se trouvent, d'en dresser des mémoires exacts et de les luy envoyer avec des échantillons. SAR jugera par elle même de la qualité des découvertes et chargera l'Académie royale des Sciences de les

de plantes marines molles ou de quelque autre matierre, qui deffendroit l'écorce du corail contre les frotements du baril et, s'il étoit possible, on en chargeroit le courier le même jour. ॥ (ibid., "I Aix-en-Provence ॥, doc. 21).

34 En 1717, par exemple, Jean-Charles Castan, bourgeois de Metz, et le chevalier de Châteaufur, ancien garde de la marine de Brest, qui ont reçu des privilèges en 1704 pour convertir le fer en acier, en demandent le renouvellement : l'enquête est toujours en cours et Réaumur a l'intention d'utiliser les informations réunies pour faire avancer ses propres recherches sur le fer et l'acier. Le Régent refuse donc de leur accorder un nouveau privilège avant que Réaumur ait terminé (document non publié, se trouvant dans le carton 16 de l'enquête, $\left.\mathrm{n}^{\circ} 16 / 03\right)$.

35 Arch. Académie des sciences, pochette de séances, 1716. C. Demeulenaere-Douyère et D. J. Sturdy, L'enquête du Régent, op. cit. note 4, "Les origines de l'enquête ", doc. 1. 


\section{L'enquête du Régent, 1716-1718}

perfectionner et de les appliquer à l'usage, ou du moins à l'instruction de la Nation. ॥

La lettre circulaire détaille les principaux sujets sur lesquels les intendants doivent s'attacher à recueillir des informations; elle leur demande également de collecter des échantillons et de les envoyer à l'Académie des sciences qui les examinera et les soumettra à des analyses.

À l'Académie royale des sciences, Réaumur est la véritable cheville ouvrière de l'enquête, même s'il se fait parfois aider par d'autres académiciens (on trouve cités les noms de Lémery et de Geoffroy). C'est lui qui prépare le travail d'enquête, en faisant établir des extraits des mémoires rédigés à la fin du siècle précédent pour l'instruction du duc de Bourgogne. \|l les étudie et les annote soigneusement. II dépouille également la plume à la main des ouvrages de référence pour rassembler des renseignements dont il demandera ensuite vérification ${ }^{36}$. Dès décembre 1715 , il est en état de formuler précisément ses premières questions.

C'est aussi Réaumur qui examine les mémoires envoyés à Paris, avec un soin remarquable et une patience admirable. II est rare qu'un premier mémoire soit pleinement satisfaisant et Réaumur a toujours de nouvelles questions à poser ; d'ailleurs, le plus souvent, un nouveau mémoire suscite de nouvelles curiosités. Des demandes d'éclaircissements sont donc renvoyées, toujours au nom du Régent, aux intendants et ceux-ci doivent se mettre à nouveau en quête d'informations complémentaires et rédiger un nouveau mémoire, présenté souvent en colonnes, les réponses de l'intendant faisant face aux questions de l'Académie et leur répondant point par point ${ }^{37}$.

Certains mémoires sont accompagnés de dessins qui représentent des mines ou des établissements industriels. Certains dessins sont bien présentés et apportent des informations utiles complétant les mémoires. Mais d'autres sont incomplets ${ }^{38}$ ou les mesures manquent, les échelles sont erronées ou inexistantes ${ }^{39}$.

36 Par exemple, Jean-Papire Masson (ibid., "Les origines de l'enquête ", doc. 3) et Pierre Borel (ibid., "Languedoc ", doc. 12). Une étude détaillée des sources bibliographiques utilisées dans l'enquête, tant par Réaumur que par les intendants, serait sans doute riche d'enseignements sur le plan de la diffusion des recherches scientifiques et de leurs résultats.

37 Par exemple, ibid., "Navarre et Béarn », doc. 20.

38 Par exemple, ibid., « Metz », doc. 6 : " On a même oublié de donner une explication du deissein qui a été envoié et de mettre des letres aux figures. ॥

39 « Les deisseins qui représentent la manière dont on tire le fil de fer sont bien entendus et faits même avec plus de pro-
Parfois les dessinateurs privilégient l'élégance ou la beauté du dessin plutôt que l'exactitude et il arrive même que les indications des dessins contredisent celles des mémoires. Comme rien n'échappe à la vigilance de Réaumur, celui-ci exige de nouveaux dessins ${ }^{40}$, et toujours il insiste : il veut des dessins exacts, avec une échelle, et non pas des dessins "finis " 4 . Il faut souligner qu'on ne trouve aujourd'hui, parmi les documents de l'enquête, qu'une infime quantité de dessins par rapport à ce qui est annoncé dans la correspondance des intendants. Réaumur en a très certainement reçus beaucoup plus qu'on n'en trouve présentement.

En ce qui concerne les échantillons, ils sont également livrés à Réaumur pour être analysés. II fait des essais lui-même, mais en confie la plupart à un assistant, Fousjean, dont on ne sait pas grand chose sinon qu'il est ancien directeur des mines de Haute-Alsace ${ }^{42}$ et essayeur expérimenté depuis au moins quinze ans ${ }^{43}$. Si l'on se fie aux en-tête des procès-verbaux d'analyse des mines ${ }^{44}$, Fousjean fait les essais à Paris, dans «le laboratoire de l'Académie ॥, laboratoire sur lequel il conviendrait de s'interroger.

Essayer les échantillons qui arrivent presque toutes les semaines par paquets, boîtes, sacs, etc., est un gros travail qui ne va pas sans problèmes. Parfois, la quantité de matière envoyée n'est pas suffisante pour permettre de faire des essais et Réaumur ou Fousjean sont obligés d'en réclamer; il y a même des cas où l'intendant oublie d'envoyer les échantillons. D'autres fois, les échantillons qui arrivent à l'Académie portent des étiquettes erronées : un intendant a désigné une matière comme «argent», alors que les essais de Réaumur ou de Fousjean montrent que c'en

preté et de soing qu'on ne l'avoit souhaité. Mais on a oublié d'y joindre une échelle et elle nous est absolument nescesaire lorsque nous les voudrons faire réduire en d'autres mesures. ॥ (ibid., " Alençon », doc. 3).

40 D. J. Sturdy, «L'Académie royale des sciences et l'enquête de Régent de 1716-1718 ॥, op. cit. note 3, pp. 138-139.

41 Entre autres exemples: «Si près d'un des endroits où l'on cultive le pastel, il y avoit quelqu'un qui scût desinner, on demanderoit des deisseins du moulin à pastel, la disposition des endroits nescesaires pour préparer le pastel et de tous les outils qui y servent. On souhaiteroit moins des deisseins finis qu'exacts accompagnés d'une échelle, et on voudroit que les deisseins en perspective fussent accompagnés de plans et de profils. ") (C. Demeulenaere-Douyère et D. J. Sturdy, L'enquête du Régent, op. cit. note 4, "Languedoc ", doc. 10).

42 Ibid., « Navarre et Béarn ॥, doc. 10.

43 bid., "Les analyses de minerais ॥, doc. 14 (Provence).

44 bid., "Les analyses de minerais », passim. 
est une autre ; il faut donc prendre soin de vérifier systématiquement la nature véritable des matières reçues. Certains intendants font essayer des échantillons par des chimistes locaux avant de les envoyer à Paris avec les rapports de ces chimistes ${ }^{45}$, mais Réaumur décourage fermement cette pratique, car il faut que tous les échantillons soient essayés par les mêmes personnes, dans les mêmes conditions et avec des méthodes identiques. Réaumur et Fousjean traitent les échantillons aussi vite qu'ils le peuvent, mais il se produit inévitablement des retards et, en janvier 1719, ils ont achevé leurs essais sur des échantillons de minerais en provenance seulement de quatorze généralités ${ }^{46}$.

\section{Les résultats}

Trois années durant, en 1716, 1717 et 1718, l'enquête est un immense chantier qui se poursuit sous la surveillance de Bignon et de Réaumur et avec les encouragements du Régent.

Quels en sont les résultats ? Et que fera-t-on de cette enquête?

\section{Un paysage minier contrasté}

Dans certaines régions, il existe des gisements de minerais qui sont bien connus et exploités de longue date : c'est le cas particulièrement de la Bretagne, où les mines de plomb argentifère de Carnouët, Plusquellec et Le Huelgoat, dans l'évêché de Quimper, sont exploitées depuis longtemps, la fin du xve siècle pour Le Huelgoat, 1682 pour Carnouët. L'enquête apporte des informations sur leurs exploitants successifs, sur la situation des gisements, la nature du minerai, sa qualité, sur la façon aussi de le transformer... Un ingénieur, Charles Thévenon, en fait une visite détaillée dont on retrouve le rapport ${ }^{47}$.

En Alsace aussi, l'exploitation des mines semble très organisée avec un important centre minier (argent et cobalt) autour de Sainte-Marie-aux-Mines qui fait l'objet de nombreux mémoires, accompagnés de très beaux dessins. L'enquête se fait l'écho de la fermeture récente, pour cause d'inondations, des mi-

45 Ibid., "Les analyses de minerais 》, doc. 3 (Languedoc). 46 La liste est la suivante: Alsace (9 mines), Bourgogne (4) Languedoc, Toulouse et Montpellier (8), Dauphiné (26), Auvergne (2), Limoges (3), Duché de Bourgogne (1), Poitiers (2), Perpignan pour le Roussillon (1), Lyon (9), Châlons pour la Champagne (1), Bretagne (6), Béarn et Basse-Navarre (25), Aix pour le Provence (7) (D. J. Sturdy, «L'Académie des sciences et l'enquête du Régent de 1716-1718॥, op. cit. note 3, p. 139-140).

47 C. Demeulenaere-Douyère et D. J. Sturdy, L'enquête du Régent, 1716-1718, op. cit. note 4, «Bretagne », doc. 7. nes d'argent de Giromagny, Lepuix et Hauxelle Haut : la communauté des mineurs de ces localités s'adresse au Régent pour dénoncer cet abandon et la destruction des mines, qui les privent, injustement selon eux, de leur travail et de leurs moyens d'existence, et leur placet provoque la mise à l'étude d'un voyage de deux académiciens sur place.

En revanche, dans beaucoup d'autres régions, l'exploitation se fait de façon plus empirique : ainsi, tout le long des Pyrénées, où il existe de très nombreux gisements de minerais divers (plomb, cuivre, fer), mais aussi de marbre, ambre, améthyste, jayet, amiante, etc., l'exploitation se fait à partir de simples excavations dans la montagne, qu'on abandonne quand la veine semble tarie. L'intendant de Perpignan « qui a tourné toute la montagne, a compté jusques à douze trous qui parroissent de douze à quinze toises de superficie de long ». Dans cette généralité, un capitaine au Royal Infanterie de Roussillon, Vilarojas, fait une visite très approfondie des pentes du mont Canigou pour y relever « les différentes mines qui y ont esté anciennement ouvertes et autres endroits où il y a lieu de croire qu'il s'en trouvera depuis $॥$, dont il dresse un rapport complet et circonstancié.

À la lecture de l'enquête, il semble que, dans beaucoup d'endroits, il y a eu à certaines époques exploitation minière, mais que souvent les trous ont été rebouchés, pour des raisons diverses, et un des objectifs de l'enquête est de faire réapparaître cette mémoire « enfouie » (et enfuie ?).

\section{Des minerais d'une grande diversité}

L'éventail des richesses minières mises en évidence par l'enquête est très large : fer, et aussi plomb et cuivre, charbon de terre, ambre, jayet (alors très utilisé dans la bijouterie de deuil), alun, amiante, mais également ardoisières, carrières de marbre (dans les Pyrénées), de pierres à bâtir diverses, de craie, de meules à aiguiser (en Alsace) ou de meules de moulin (à La Ferté-sous-Jouarre)... Des pierres précieuses aussi, ou non précieuses : turquoises (dans le Gers), améthystes, jacinthes, " cailloux » transparents de couleurs (en Auvergne), etc. Mais il est évident que ce sont les métaux précieux qui intéressent avant tout les enquêteurs : l'or et l'argent, sous forme de filons ou de paillettes roulées par les rivières (dans le Languedoc, en Alsace, en Lyonnais) ; les informateurs devinent aisément cette attente et «révèlent » des mines d'or oubliées comme la mine de Bouconville, dans le Soissonnais, ou des filons perdus dans la montagne comme la mine de Bourg-d'Oisans, en Dauphiné. 


\section{L'enquête du Régent, 1716-1718}

\section{Un tableau du monde minier}

L'enquête veille à resituer l'exploitation minière dans son contexte, depuis la localisation des filons, leur accessibilité, leur environnement (existence à proximité de forêts, de cours d'eau...) jusqu'aux industries de transformation qui leur sont étroitement liées : fourneaux pour fondre le minerai, forges, moulins pour tailler les pierres précieuses, etc., qui font l'objet de descriptions minutieuses et dont on trouve quelques dessins, comme particulièrement pour Sainte-Marieaux-Mines.

À travers l'enquête, apparaissent aussi les mineurs, en groupe comme ceux de la communauté des mineurs de Giromagny qui protestent contre l'abandon de leurs mines, ou individuellement. Dans ce dernier cas, ils sont surtout perçus comme des "producteurs » et ce sont leurs " attitudes » de travail qui intéressent avant tout l'Académie des sciences et Réaumur ; en revanche, jamais les menaces qui pèsent sur eux dans l'exécution de leur travail journalier ne sont évoquées ni par les intendants ou leurs informateurs, ni par l'Académie.

Dans l'optique du projet des Descriptions des arts et métiers, Réaumur est très attentif au « comment faiton ? ॥. Les outils l'intéressent au plus haut point, qu'il s'agisse des planches dont se servent les laveurs d'or ou des outils utilisés pour extraire les minerais, et il en réclame toujours des dessins « exacts » et détaillés ${ }^{48}$.

L'enquête de 1716-1718, aussi élaborée et complète qu'elle ait été, n'eut pas de suites immédiates et ses résultats restèrent dans les cartons. La disparition du Régent, en 1723, semble l'avoir poussée dans l'oubli administratif ; en effet, la mort rapide de celui qui en avait été le promoteur et le principal soutien, fit passer ses résultats à l'arrière-plan des préoccupations politiques de ses successeurs au gouvernement, et elle n'a pas débouché immédiatement, comme on aurait pu l'attendre, sur une politique de mise en valeur des ressources naturelles du royaume.

Réaumur, qui était chargé de la conduite scientifique de l'enquête, a utilisé des informations collectées à cette occasion pour certains de ses travaux personnels ou pour la documentation de son Art de convertir le fer forgé en acier, et l'art d'adoucir le fer fondu (1722), mais il semble se détourner assez rapidement de l'enquête au profit d'autres intérêts scientifiques.

Quant aux Descriptions des arts et métiers, qui sont relancées sous la nouvelle direction de Duhamel du Monceau après la mort de Réaumur en 1757, elles ne peuvent plus alors tirer un bénéfice immédiat d'informations qui avaient toute leur valeur et leur actualité dans les années 1719 et suivantes, mais qui, quarante ans plus tard, sont devenues en partie au moins obsolètes.

Cependant, si l'enquête de 1716-1718 peut apparaître comme une tentative restée inachevée, elle marque néanmoins une étape importante dans l'élaboration d'un mode de collaboration entre l'État et l'Académie royale des sciences. Par ailleurs, elle a concouru à habituer les administrateurs du royaume (en l'occurrence les intendants) à prendre en compte des critères « scientifiques » ou « techniques » dans la gestion, notamment économique, des territoires qui leur étaient confiés et, en ce sens, elle participe à l'invention de méthodes modernes d'administration. E† on peut voir comme un prolongement plus lointain de cet intérêt pour le monde minier et ses ressources qu'elle a révélé, dans la mise en place, à partir du milieu du xvIII siècle, d'une administration des mines plus structurée.

48 Sur le rôle que Réaumur assigne au dessin dans l'enquête du Régent, C. Demeulenaere-Douyère, "Enquête administrative et dessin technique dans la France pré-industrielle... », op. cit. note 7. 